\title{
Linear Response Theory and Green-Kubo Relations for Active Matter
}

\author{
Sara Dal Cengio $\odot,{ }^{1}$ Demian Levis, ${ }^{1,2,3,{ }^{*}}$ and Ignacio Pagonabarraga ${ }^{1,2,3}$ \\ ${ }^{1}$ Departament de Física de la Matèria Condensada, Universitat de Barcelona, Martí i Franquès 1, E08028 Barcelona, Spain \\ ${ }^{2}$ CECAM Centre Européen de Calcul Atomique et Moléculaire, École Polytechnique Fédérale de Lausanne, \\ Batochime, Avenue Forel 2, 1015 Lausanne, Switzerland \\ ${ }^{3}$ UBICS University of Barcelona Institute of Complex Systems, Martí i Franquès 1, E08028 Barcelona, Spain
}

(Received 5 July 2019; published 3 December 2019)

\begin{abstract}
We address the question of how interacting active systems in a nonequilibrium steady state respond to an external perturbation. We establish an extended fluctuation-dissipation theorem for active Brownian particles (ABP), which highlights the role played by the local violation of detailed balance due to activity. By making use of a Markovian approximation we derive closed Green-Kubo expressions for the diffusivity and mobility of $\mathrm{ABP}$ and quantify the deviations from the Stokes-Einstein relation. We compute the linear response function to an external force using unperturbed simulations of ABP and compare the results with the analytical predictions of the transport coefficients. Our results show the importance of the interplay between activity and interactions in the departure from equilibrium linear response.
\end{abstract}

DOI: 10.1103/PhysRevLett.123.238003

Linear response theory describes how a (small) external perturbation affects the macroscopic properties of a system [1]. If the unperturbed system is initially in equilibrium, its response to perturbations is generically related to equilibrium fluctuations through the fluctuation-dissipation theorem (FDT). In this situation, one can derive exact expressions for the transport coefficients in terms of equilibrium correlations, the so-called Green-Kubo relations, which are among the very rare general results in nonequilibrium statistical mechanics [2]. However, intrinsically nonequilibrium systems, such as active matter, lie beyond the scope of this framework. Indeed, active matter stands for systems made of components which typically convert energy from their environment into motion in a way that breaks detailed balance [3-6]. Therefore, equilibrium states cannot be considered as the reference unperturbed states in that case.

Extensions of the FDT to nonequilibrium states have been recently derived [7-12]. However, these approaches have not been applied to locally driven active systems and general Green-Kubo-like expressions, relating their transport coefficients with nonequilibrium steady-state correlations, have not been established yet. Establishing the nature of these relationships has deep consequences, because of the insight they provide in the nature of nonequilibrium response in active matter. For example, experiments on microswimmer suspensions have revealed interesting rheological behavior $[13,14]$ and rectification phenomena in the presence of asymmetric boundaries [15-17], opening up the possibility to exploit the nonequilibrium character of active matter to control transport at the microscale and extract energy from it. Harvesting the potential of these systems thus needs the development of an extended response theory: This is the overall aim of the present work.
We start by considering a generic time evolution of the probability density $\Psi(\Gamma, t)$ to find the system in a certain configuration $\Gamma$ of the phase space:

$$
\partial_{t} \Psi=\Omega \Psi
$$

At $t=0$ we perturb the system and follow its evolution. The generator of the dynamics $\Omega=\Omega_{0}+\Omega_{\text {ext }}$ can be split into an unperturbed $\Omega_{0}$ and perturbed $\Omega_{\text {ext }}$ part. For $t<0$ the system is in its steady state $\Psi_{0}$, defined by $\Omega_{0} \Psi_{0}=0$ [18]. Using the operator identity $e^{\Omega t}=1+\int_{0}^{t} d t^{\prime} e^{\Omega t} \Omega$, we can derive the following expression of the average of an observable $A$ at any time $t \geq 0,\langle A\rangle_{t}=\int d \Gamma \Psi(\Gamma, t) A(\Gamma)$, as

$$
\langle A\rangle_{t}-\langle A\rangle_{0}=\int_{0}^{t} d s\left\langle\frac{\Omega_{\mathrm{ext}} \Psi_{0}}{\Psi_{0}} A(s)\right\rangle_{0}
$$

Here $\langle *\rangle_{0}$ denotes averages over $\Psi_{0}$, while $A(s)=e^{\Omega^{\dagger} s} A(0)$, where $\Omega^{\dagger}$ is the adjoint (backwards) Smoluchowski operator [20] (in the Heisenberg representation of the ensemble average). Note that while the former expression is completely general and does not rely on any perturbation expansion (therefore also valid in the non linear regime), its application demands some knowledge of the distribution $\Psi_{0}$, or at least the action of $\Omega_{\text {ext }}$ on it $[8,12,21,22]$.

For the sake of clarity, let us focus first on an equilibrium system of $(N+1)$ overdamped Brownian particles, interacting by means of potential forces $\boldsymbol{F}_{i}=-\partial_{i} U\left(\partial_{i}\right.$ denotes the spatial gradient $\partial / \partial \boldsymbol{r}_{i}$ ) with diffusion coefficient $D_{0}$, mobility $\mu_{0}$, and inverse temperature $\beta=\mu_{0} / D_{0}$. In that case, Eq. (1) corresponds to the Smoluchowski equation describing the time evolution of the probability density of a 
point in configuration space $\Gamma \equiv\left(\boldsymbol{r}_{1}, \ldots, \boldsymbol{r}_{N}, \boldsymbol{r}_{n}\right)$, with $\Omega_{0} \equiv \Omega_{\mathrm{eq}}=\sum_{i}\left[\partial_{i} \cdot\left(D_{0} \partial_{i}-\mu_{0} \boldsymbol{F}_{i}\right)\right]$. We perturb an initially equilibrated system $\left(\Psi_{0} \equiv \Psi_{\mathrm{eq}} \sim e^{-\beta U}\right)$ by applying a constant force $\boldsymbol{f}=\boldsymbol{f} \boldsymbol{u}_{x}$ to a tracer particle described by $\boldsymbol{r}_{n}$; thus, $\Omega_{\text {ext }}=-\mu_{0} f \cdot \partial_{n}$ and

$$
\Omega_{\mathrm{ext}} \Psi_{\mathrm{eq}}=-\beta \mu_{0} \boldsymbol{f} \cdot \boldsymbol{F}_{n} \Psi_{\mathrm{eq}} \cdot
$$

From Eq. (2) we find

$$
\langle A\rangle_{t}-\langle A\rangle_{0}=-\beta \mu_{0} f \int_{0}^{t} d s\left\langle\boldsymbol{F}_{n} e^{\Omega^{\dagger} s} A\right\rangle_{0} .
$$

This equation constitutes a generalized nonlinear GreenKubo expression, relating a nonequilibrium average at time $t$ with an equilibrium time correlation. Such an approach, originally introduced in the context of glassy rheology, is usually referred to as integration through transients (ITT) [23-29]. If we now choose $A \equiv F_{n}^{x}=\boldsymbol{F}_{n} \cdot \boldsymbol{u}_{x}$ in Eq. (4), and define the tracer mobility $\mu \equiv \lim _{t \rightarrow \infty}\left\langle\dot{\boldsymbol{r}}_{n}\right\rangle / \boldsymbol{f}$, we find, in the linear regime $f \rightarrow 0$, the standard GreenKubo relation: $\mu=\beta \int_{0}^{t} d t^{\prime}\left\langle\dot{x}_{n}\left(t^{\prime}\right) \dot{x}_{n}(0)\right\rangle_{0}=\beta D$ (see the Supplemental Material [30]).

We consider now active Brownian particles (ABP) [31], self-propelled with a constant velocity $v_{0}$ in the 2D plane along their orientation $\boldsymbol{n}_{i}=\left(\cos \theta_{i}, \sin \theta_{i}\right)$. The dynamic equations read

$$
\dot{\boldsymbol{r}}_{i}(t)=\mu_{0} \boldsymbol{F}_{i}+v_{0} \boldsymbol{n}_{i}(t)+\boldsymbol{\xi}_{i}(t), \dot{\theta}_{i}(t)=\zeta_{i}(t),
$$

where $\boldsymbol{F}_{i}$ accounts for all interparticle potential forces (typically short-range repulsions) and $\mu_{0}$ is the mobility. The noise terms, $\boldsymbol{\xi}_{i}$ and $\zeta_{i}$ are Gaussian and white, with zero mean and variance $2 D_{0}$ and $2 D_{\theta}$, with $D_{0}=\mu_{0} / \beta$ the thermal Brownian diffusivity and $D_{\theta}$ the rotational diffusion coefficient, introducing the persistence time $\tau=1 / D_{\theta}$. Equilibrium is recovered both in the limit of $v_{0} \rightarrow 0$ or $\tau \rightarrow 0$. The ABP system above is one of the reference models for active matter, and it has been studied extensively. ABP has been characterized in terms of thermodynamic quantities such as pressure and chemical potential [32-37], and its phase behavior analyzed in great detail [38-43]. Several recent works have studied its linear response (and of similar active particles modeled in terms of an Ornstein-Uhlenbeck process) from different viewpoints: (i) introducing an effective temperature characterizing FDT violations [44-47]; (ii) taking equilibrium as the reference state and considering activity as a perturbation of it $[28,29,48]$; (iii) deriving expressions of linear response functions in terms of weighted averages over the unperturbed dynamics $[47,49,50]$, in the same spirit as the Malliavin weights sampling [51]. In the present Letter, we first characterize the violations of the FDT in ABP, showing how the nonequilibrium character of activity comes into play in an extended FDT that we establish, and then derive Green-Kubo expressions for its transport coefficients in terms of its nonequilibrium fluctuations.

We first analyze how ABP (in a nonequilibrium steady state) respond to an external perturbation. Our starting point is the Smoluchowski operator corresponding to Eq. (5),

$$
\Omega_{0}=\sum_{i}\left(\partial_{i} \cdot\left(D_{0} \partial_{i}-\mu_{0} \boldsymbol{F}_{i}-v_{0} \boldsymbol{n}_{i}\right)+D_{\theta} \frac{\partial^{2}}{\partial \theta_{i}^{2}}\right),
$$

with a perturbation due to an external force $f=-\partial_{n} V$, where $V=-f x_{n}$, applied to a tagged particle located in $\boldsymbol{r}_{n}=\left(x_{n}, y_{n}\right)$. A salient feature of ABP is the absence of zero-flux steady-state solutions of the Smoluchowski equation (which can be seen from the impossibility to simultaneously satisfy the zero-current conditions for the angular and positional degrees of freedom). In the absence of odd variables under time reversal, as is the case for this overdamped dynamics, the absence of a zero-flux solution is a necessary and sufficient condition for the violation of detailed balance [52]. Even free ABP have nonzero fluxes in the steady state (corresponding to their self-propulsion velocity, see Ref. [30]). This is in contrast with continuum descriptions of active matter which can recover detailed balance after coarse-graining [53-55] and to active OrnsteinUhlenbeck particles (AOUP), which fulfill detailed balance in the presence of potentials with zero third derivatives $[48,56]$ (see the Supplemental Material [30]).

Since the stationary probability density must be positive, normalizable and differentiable, we may write

$$
\Psi_{0}(\Gamma) \sim e^{-\beta\left[U\left(\left\{\boldsymbol{r}_{i}\right\}\right)+v_{0} \tau \Xi\left(\left\{\boldsymbol{r}_{i}, \theta_{i}\right\}\right)\right]}
$$

in terms of a generalized potential $v_{0} \tau \Xi\left(\left\{\boldsymbol{r}_{j}, \theta_{j}\right\}\right)$ [57] encoding deviations from Boltzmann statistics. The steady state condition $\Omega_{0} \Psi_{0}=0$ leads to

$$
v_{0} \partial_{i} \cdot\left(-\mu_{0} \tau \partial_{i} \Xi-\boldsymbol{n}_{i}\right) \Psi_{0}+\tau^{-1} \partial_{\theta_{i}}^{2} \Psi_{0}=-\nabla_{i} \cdot \boldsymbol{j}_{i},
$$

where $\nabla_{i}=\left(\partial_{i}, \partial_{\theta_{i}}\right)$, introducing the steady-state local velocity

$$
\boldsymbol{\nu}_{i} \equiv \boldsymbol{j}_{i} / \Psi_{0}=v_{0}\left[\left(\boldsymbol{n}_{i}+\mu_{0} \tau \partial_{i} \Xi\right), \beta \partial_{\theta_{i}} \Xi\right] .
$$

In order to make the connection between the steady-state current and the local violation of detailed balance explicit, we consider the time-reversed adjoint operator $\bar{\Omega}_{0}^{\dagger}$ [defined as $\left.A(-t)=e^{\bar{\Omega}_{0}^{\dagger} t} A(0)\right]$ and find [30]

$$
\Omega_{0}^{\dagger}-\bar{\Omega}_{0}^{\dagger}=2 \mathcal{V} \cdot \boldsymbol{\nabla}
$$

where $\mathcal{V}=\left\{\boldsymbol{\nu}_{i}\right\}$ and $\boldsymbol{\nabla}=\left\{\partial_{i}, \partial_{\theta_{i}}\right\}$. Equation (10) shows that violations of detailed balance and nonzero steady current are two faces of the same coin. Note that violations of detailed balance can be quantified using time-reversed 
trajectories $[22,48,58]$. In the passive limit $v_{0} \rightarrow 0$ detailed balance is recovered and $\boldsymbol{\nu}_{i} \rightarrow 0$.

Inserting Eq. (7) into Eq. (2) yields

$$
\begin{aligned}
& \langle A\rangle_{t}-\langle A\rangle_{0} \\
& =\beta\left(\int_{0}^{t} d s\langle\dot{V}(0) A(s)\rangle_{0}-\int_{0}^{t} d s\langle\mathcal{V} \cdot \nabla V(0) A(s)\rangle_{0}\right) .
\end{aligned}
$$

In the linear regime, the latter expression constitutes an extension of the FDT far from equilibrium $[7,8]$. When $\mathcal{V}=0$ one easily recovers the standard FDT. Activity is responsible for the second term $\propto \mathcal{V}$ in Eq. (11), which quantifies the local dissipation of energy required to maintain the nonequilibrium steady state [59]. For free ABP (for which $\boldsymbol{\nu}_{i}=v_{0} \boldsymbol{n}_{i}$ ), the correction to the FDT can be recast in terms of an effective temperature (see the Supplemental Material [30]).

As opposed to the equilibrium FDT, the response of an active system is not completely determined by its fluctuations, but depends on the specific form of its steady-state distribution $[12,20,48]$. Our aim being to derive explicit Green-Kubo relations for ABP (which do not depend on an unknown generalized potential), we forbid ourselves to make any assumption about steady-state properties but only rely on the dynamics. Our starting point should thus be Eq. (5), although for this dynamics we cannot derive an analog of Eq. (3). Thus, the ITT construction that leads us to Eq. (4) cannot be readily followed. To overcome this difficulty we integrate out the angular variables and work with the following reduced dynamics [38,41]:

$$
\dot{\boldsymbol{r}}_{i}(t)=\mu_{0} \boldsymbol{F}_{i}+\boldsymbol{\eta}_{i}(t),
$$

where the noise $\boldsymbol{\eta}_{i}$ is approximately Gaussian [60] with zero mean and variance $\left\langle\boldsymbol{\eta}_{i}(t) \boldsymbol{\eta}_{j}(s)\right\rangle=\left[2 D_{0} \delta(t-s)+\right.$ $\left.v_{0}^{2} e^{-|t-s| / \tau} / 2\right] \delta_{i j} 1$ [61]. As usual, by integrating away some degrees of freedom one generates memory, here with a time correlation $\tau \equiv 1 / D_{\theta}$. Even if in the noninteracting limit particles described by Eq. (12) diffuse at long times with a diffusivity $D_{a}=D_{0}+v_{0}^{2} \tau / 2$, the difficulty resides on the non-Markovianity of the evolution, which cannot be formulated in terms of a Smoluchowski operator; a longstanding problem in statistical mechanics [62-65]. Fox developed a first order in $\tau$ expansion $[65,66]$ that leads to an effective Smoluchowski equation and which has proven useful in the context of ABP and AOUP [41,67]. Equation (12) then reduces to $[41,65,66]$

$$
\Omega_{0}^{M}=\sum_{i=1}^{N} \partial_{i} \cdot \mathcal{D}_{i}(\Gamma)\left[\partial_{i}-\beta \boldsymbol{F}_{i}^{\mathrm{eff}}(\Gamma)\right],
$$

where we have introduced an effective diffusivity and interaction force

$$
\begin{aligned}
\mathcal{D}_{i}(\Gamma) & =D_{0}+\frac{v_{0}^{2} \tau}{2}\left(1+\frac{\tau \mu_{0} \partial_{i} \cdot \boldsymbol{F}_{i}}{1-\tau \mu_{0} \partial_{i} \cdot \boldsymbol{F}_{i}}\right), \\
\boldsymbol{F}_{i}^{\mathrm{eff}}(\Gamma) & =\left(D_{0} \boldsymbol{F}_{i}-\beta^{-1} \partial_{i} \mathcal{D}_{i}\right) / \mathcal{D}_{i} .
\end{aligned}
$$

(Note that $\tau \mu_{0} \partial_{i} \cdot \boldsymbol{F}_{i}<1$ must be ensured.) The dynamics encoded in Eq. (13) fulfills detailed balance. The nonequilibrium character of the problem is now encoded in the effective diffusivity (which now depends on the relative positions of all the particles) [68-70] and forces (which do not derive from a potential) rather than on the (lack of) symmetries of the dynamics. Equation (13) corresponds to an effective equilibrium description, for which the steadystate distribution is non-Boltzmann but has zero current. As we show below, such first-order expansion provides relevant and systematic deviations to equilibrium linear response. A higher order expansion would not improve the present approximation, as an effective Smoluchowskilike description [such as Eq. (13)] for a non-Markovian dynamics can only exist to first order in $\tau[62,71]$. For a constant force perturbation we find

$$
\Omega^{M} \Psi_{0}=\left(\Omega_{0}^{M}+\Omega_{\mathrm{ext}}\right) \Psi_{0}=-\beta \mu_{0} \boldsymbol{f} \cdot \boldsymbol{F}_{n}^{\mathrm{eff}} \Psi_{0},
$$

which allows us to derive an analog of Eq. (4),

$$
\langle A\rangle_{t}-\langle A\rangle_{0}=-\mu_{0} \beta \boldsymbol{f} \cdot \int_{0}^{t} d s\left\langle\boldsymbol{F}_{n}^{\mathrm{eff}} e^{\left(\Omega^{M}\right)^{\dagger} s} A\right\rangle_{0} .
$$

Equation (17) allows us to derive Green-Kubo expressions of the diffusivity and mobility which do not rely of the Stokes-Einstein relation, but only on the time evolution of the system under the Markovian approximation Eq. (14). By choosing $A \equiv F_{n}^{x}$ in Eq. (17) we get the following Green-Kubo relation for the mobility

$$
\mu=\mu_{0}\left(1-\mu_{0} \beta \int_{0}^{\infty} d s\left\langle F_{n}^{\mathrm{eff}, x}(0) F_{n}^{x}(s)\right\rangle_{0}\right),
$$

which is identical to what one would get in the passive case by replacing $F_{n}^{\text {eff }}$ by $F_{n}$. To first order in $\tau \mu_{0} \partial_{i} \cdot \boldsymbol{F}_{i}$, it reads

$$
\begin{aligned}
\frac{\mu}{\mu_{0}}= & 1-\frac{\mu_{0}^{2}}{D_{a}} \int_{0}^{\infty} d s\left\langle F_{n}^{x}(0) F_{n}^{x}(s)\right\rangle_{0} \\
& +\frac{v_{0}^{2} \tau^{2} \mu_{0}^{3}}{2 D_{a}^{2}} \int_{0}^{\infty} d s\left\langle F_{n}^{x}(0) \partial_{n} \cdot \boldsymbol{F}_{n}(0) F_{n}^{x}(s)\right\rangle_{0} \\
& +\frac{v_{0}^{2} \tau^{2} \mu_{0}^{2}}{2 D_{a}} \int_{0}^{\infty} d s\left\langle\partial_{n}^{x} \partial_{n} \cdot \boldsymbol{F}_{n}(0) F_{n}^{x}(s)\right\rangle_{0} .
\end{aligned}
$$

For passive systems, only the terms in the first line survive, capturing how interactions affect the ideal gas mobility. Here, activity plays a role in the statistics of collisions, thus the force self-correlation function, and in the value of the prefactor via the single particle diffusivity. The remaining 
two terms $\propto\left(v_{0} \tau\right)^{2}$ correspond to subdominant higherorder correlations involving many-body interactions.

To characterize the departure from equilibrium linear response in terms of the Stokes-Einstein relation, we use (see Ref. [30] for a proof)

$$
\mu_{0}^{2}\left\langle F_{n}^{x}(0) \cdot F_{n}^{x}(t)\right\rangle_{0}=\left\langle\mathcal{D}_{n}\right\rangle_{0} \delta(t)-\left\langle\dot{x}_{n}(0) \dot{x}_{n}(t)\right\rangle_{0},
$$

which relates the force and the velocity autocorrelation functions, and whose functional form depends only on the properties of the evolution operator (for equilibrium Brownian dynamics it was derived in Ref. [72]). Once we identify the diffusivity with the velocity self-correlation function, we get the Green-Kubo expression

$$
\begin{aligned}
D= & D_{a}+\frac{\mu_{0} v_{0}^{2} \tau^{2}}{2}\left\langle\partial_{n} \cdot \boldsymbol{F}_{n}\right\rangle_{0} \\
& -\mu_{0}^{2} \int_{0}^{\infty} d s\left\langle F_{n}^{x}(0) F_{n}^{x}(s)\right\rangle_{0},
\end{aligned}
$$

which allows us to express Eq. (19) as

$$
\frac{\mu}{\mu_{0}}=\frac{D}{D_{a}}-\frac{\mu_{0} v_{0}^{2} \tau^{2}}{2 D_{a}}\left\langle\partial_{n} \cdot \boldsymbol{F}_{n}\right\rangle_{0}+\text { h.o.t. }
$$

where h.o.t. refers to higher order terms. Equation (22) reduces to the usual Stokes-Einstein relation in the passive limit. If $\partial_{n} \cdot \boldsymbol{F}_{n}=0$, a modified Stokes-Einstein relation holds, $\mu=\beta_{\text {eff }} D$, with an effective temperature $k_{B} T_{\text {eff }}=$ $D_{a} / \mu_{0}[45-47,73]$. Indeed, genuine nonequilibrium behavior results from the combined effect of interactions and activity, as observed in active colloidal suspensions [74] and proven for AOUP $[48,56,58]$ (among other examples).

To illustrate our results and put them into test, we run particle-based simulations of ABP Eq. (5) with periodic boundary conditions. We consider the pair potential $U(r)=(\sigma / r)^{12}$ and the following set of parameters: $\tau=0.02, \mu_{0}=1$, and $\beta=1$. We vary the Peclet number $\mathrm{Pe}=v_{0} \tau / \sigma$ and the mean density $\phi=\left(\pi \sigma^{2} N / 4 L^{2}\right)$ in a range for which the system remains homogeneous $[\mathrm{Pe} \in(0: 10)$ and $\phi \in(0.01: 0.2)]$.

We analyze the integrated response $\chi(t)=$ $N^{-1} \lim _{f \rightarrow 0} \sum_{i}^{N}\left[\left\langle x_{i}(t)-x_{i}(0)\right\rangle\right] / f$ of the particles' positions due to a constant force applied to all of them $\boldsymbol{f}_{i}=\epsilon_{i} f \boldsymbol{u}_{x}$, where $\epsilon_{i}= \pm 1$ with equal probability [45]. We compute $\chi$ using two different strategies: (i) we explicitly apply a small force and measure the particle displacements it induces; (ii) we track the appropriate stochastic variables needed to compute the response function of interest using simulations of the unperturbed dynamics. The first "direct method" involves computing displacements generated by a small perturbation that guarantees the linear regime. The second "Malliavin weight (MW) method" overcomes the considerable numerical uncertainties (and cost) related to the control of a small perturbation parameter in Brownian
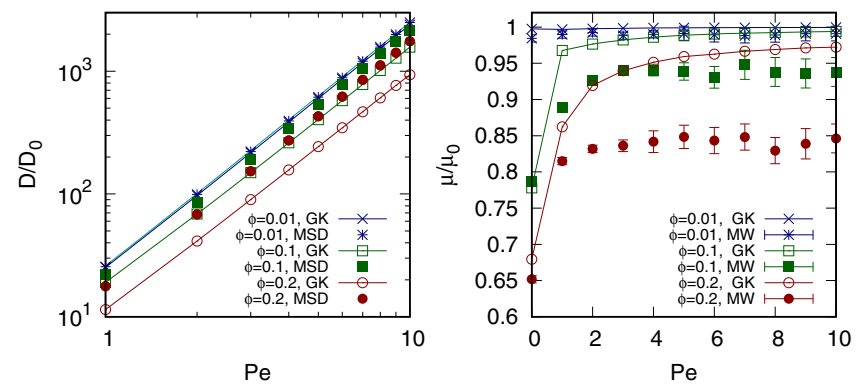

FIG. 1. Transport coefficients for ABP at $\phi=0.01,0.1$, and 0.2 as a function of Pe. Left: Diffusion coefficient obtained from the long-time behavior of the mean-squared displacement (MSD, with points) compared to the Green-Kubo expression Eq. (21) (GK, with line points). Right: Mobility obtained from simulations of unperturbed ABP using Malliavin weights (MW, with error bars) compared to the Green-Kubo prediction Eq. (19) (GK, with point lines).

dynamics simulations; we thus make an extensive use of it in the following. This method, originally introduced in the context of Monte Carlo simulations of spin systems [75], and then generalized to Brownian dynamics [51,76], was recently extended to active particles $[47,49]$.

We compute $D=N^{-1} \lim _{t \rightarrow \infty} \sum_{i}\left\langle\left[\boldsymbol{r}_{i}(t)-\boldsymbol{r}_{i}(0)\right]^{2}\right\rangle /(4 t)$ from the long-time behavior of the mean-square displacement (MSD). According to the MW method, the response function of interest can be expressed as [47,51]

$$
\chi(t)=N^{-1} \sum_{i}\left\langle x_{i}(t) q_{i}(t)\right\rangle,
$$

where $q_{i}$ is a Malliavin weight that evolves accordingly to $\dot{q}_{i}(t)=\sqrt{\beta / 2} \sum_{i} \xi_{i}^{x}(t)$, and averages are taken over independent realizations of the unperturbed dynamics. We thus compute $\chi$ using Eq. (23) and extract $\mu=\lim _{t \rightarrow \infty} \chi(t) / t$ (after checking consistency with the direct method, see the Supplemental Material [30]). We also compute $D$ and $\mu$ using our Green-Kubo expressions. To be concrete we compute, from simulations of $\mathrm{ABP}$, the different terms involving correlations and gradients of the potential that appear in Eq. (21) and Eq. (19). The results obtained are shown in Fig. 1. The diffusion coefficient follows the same growth $\sim \mathrm{Pe}^{2}$ as the ideal gas in the parameter range explored, but decreases with $\phi$. Equation (21) underestimates the value of $D$ obtained from the MSD but retains its functional dependence. Mobility is not affected by activity in the dilute regime but decreases as the density increases, as expected from Eq. (19) and Refs. [45,47]. At finite density and $\mathrm{Pe}, \mu$ decreases with density but remains roughly constant for Pe $\gtrsim 2$. Such behavior is reproduced by Eq. (19), although it overestimates the numerical value. Our Green-Kubo expressions predict the qualitative behavior of $D$ and $\mu$, despite we cannot reach a precise quantitative agreement at high $\mathrm{Pe}$ and $\phi$. Although such mismatch, expected from the basic assumptions behind the 
approximations made (small values of $\tau$ and $\tau \mu_{0} \partial_{i} \cdot \boldsymbol{F}_{i}$; neither too active nor too dense), prevents quantitative agreement, the derived Green-Kubo expressions provide a general understanding on how the interplay between activity and interactions affects the transport properties of active particle systems.

The response of nonequilibrium systems is typically sensitive to details of the unperturbed initial state, hence the difficulty in establishing a general theory. Such lack of universality is encoded in the presence of the generalized potential in extended fluctuation-dissipation relations, arising from the breakdown of detailed balance at the microscopic level. In this Letter we set the stage for a systematic response theory of active systems obtained on pure dynamical grounds. Via a Markovian approximation scheme, valid in the limit of small persistence time and moderate density, we overcome the aforementioned difficulty and we put forward a closed Green-Kubo expression for the mobility and diffusivity. This allows us to quantify the breakdown of the Stokes-Einstein relation due to the interplay between activity and interparticle interactions. Extending the present approach to deal with different selfpropulsion or interaction mechanisms and other transport coefficients such as the shear viscosity [77] will then provide a theoretical framework to gain insights on the rheological properties of active matter $[13,14]$.

We warmly thank Thomas Voigtmann, Udo Seifert, Roland Netz, Jose M. Sancho, and Miguel Rubi for discussions and suggestions. S. D. C. and I. P. acknowledge funding from the European Union's Horizon 2020 program under ETN Grant Agreement No. 674979-NANOTRANS. D. L. acknowledges funding from EU Horizon 2020 program under the Marie Sklodowska-Curie Actions H2020MSCA-IF Grant Agreement No. 657517.

*demian.levis@epfl.ch

[1] U. M. B. Marconi, A. Puglisi, L. Rondoni, and A. Vulpiani, Phys. Rep. 461, 111 (2008).

[2] R. Kubo, M. Toda, and N. Hashitsume, Statistical Physics II: Nonequilibrium Statistical Mechanics (Springer Science \& Business Media, New York, 1991), Vol. 31.

[3] M. C. Marchetti, J.-F. Joanny, S. Ramaswamy, T. B. Liverpool, J. Prost, M. Rao, and R. A. Simha, Rev. Mod. Phys. 85, 1143 (2013).

[4] M. E. Cates, Rep. Prog. Phys. 75, 042601 (2012).

[5] É. Fodor and M. C. Marchetti, Physica (Amsterdam) 504A, 106 (2018).

[6] C. Battle, C. P. Broedersz, N. Fakhri, V. F. Geyer, J. Howard, C. F. Schmidt, and F. C. MacKintosh, Science 352, 604 (2016).

[7] M. Baiesi, C. Maes, and B. Wynants, Phys. Rev. Lett. 103, 010602 (2009).

[8] M. Baiesi and C. Maes, New J. Phys. 15, 013004 (2013).

[9] R. Chetrite, G. Falkovich, and K. Gawedzki, J. Stat. Mech. (2008) P08005.
[10] J. Prost, J.-F. Joanny, and J. M. R. Parrondo, Phys. Rev. Lett. 103, 090601 (2009).

[11] U. Seifert and T. Speck, Europhys. Lett. 89, 10007 (2010).

[12] T. Speck and U. Seifert, Europhys. Lett. 74, 391 (2006).

[13] H. M. López, J. Gachelin, C. Douarche, H. Auradou, and E. Clément, Phys. Rev. Lett. 115, 028301 (2015).

[14] S. Rafaï, L. Jibuti, and P. Peyla, Phys. Rev. Lett. 104, 098102 (2010).

[15] R. Di Leonardo, L. Angelani, D. Dell'Arciprete, G. Ruocco, V. Iebba, S. Schippa, M. Conte, F. Mecarini, F. De Angelis, and E. Di Fabrizio, Proc. Natl. Acad. Sci. U.S.A. 107, 9541 (2010).

[16] N. Koumakis, A. Lepore, C. Maggi, and R. Di Leonardo, Nat. Commun. 4, 2588 (2013).

[17] C. Maggi, J. Simmchen, F. Saglimbeni, J. Katuri, M. Dipalo, F. De Angelis, S. Sanchez, and R. Di Leonardo, Small 12, 446 (2016).

[18] Note that the condition $\Omega_{0} \Psi_{0}=0$ only implies stationarity but not detailed balance [19].

[19] R. Graham and H. Haken, Z. Phys. A 243, 289 (1971).

[20] H. Risken, in The Fokker-Planck Equation (Springer, New York, 1996), pp. 63-95.

[21] G. Agarwal, Z. Phys. A 252, 25 (1972).

[22] D. Chaudhuri, Phys. Rev. E 90, 022131 (2014).

[23] M. Fuchs and M.E. Cates, Phys. Rev. Lett. 89, 248304 (2002).

[24] M. Fuchs and M. E. Cates, J. Phys. Condens. Matter 17, S1681 (2005).

[25] M. Fuchs and M. E. Cates, J. Rheol. 53, 957 (2009).

[26] I. Gazuz, A. M. Puertas, T. Voigtmann, and M. Fuchs, Phys. Rev. Lett. 102, 248302 (2009).

[27] I. Gazuz and M. Fuchs, Phys. Rev. E 87, 032304 (2013).

[28] A. Sharma and J. M. Brader, J. Chem. Phys. 145, 161101 (2016).

[29] A. Liluashvili, J. Ónody, and T. Voigtmann, Phys. Rev. E 96, 062608 (2017).

[30] See Supplemental Material at http://link.aps.org/ supplemental/10.1103/PhysRevLett.123.238003 for further details on the analytical derivations and numerical simulations.

[31] P. Romanczuk, M. Bär, W. Ebeling, B. Lindner, and L. Schimansky-Geier, Eur. Phys. J. Spec. Top. 202, 1 (2012).

[32] S. C. Takatori, W. Yan, and J. F. Brady, Phys. Rev. Lett. 113, 028103 (2014).

[33] A. P. Solon, J. Stenhammar, R. Wittkowski, M. Kardar, Y. Kafri, M. E. Cates, and J. Tailleur, Phys. Rev. Lett. 114, 198301 (2015).

[34] R. G. Winkler, A. Wysocki, and G. Gompper, Soft Matter 11, 6680 (2015).

[35] D. Levis, J. Codina, and I. Pagonabarraga, Soft Matter 13, 8113 (2017).

[36] A. P. Solon, J. Stenhammar, M. E. Cates, Y. Kafri, and J. Tailleur, Phys. Rev. E 97, 020602(R) (2018).

[37] S. Paliwal, J. Rodenburg, R. van Roij, and M. Dijkstra, New J. Phys. 20, 015003 (2018).

[38] Y. Fily and M. C. Marchetti, Phys. Rev. Lett. 108, 235702 (2012).

[39] G. S. Redner, M. F. Hagan, and A. Baskaran, Phys. Rev. Lett. 110, 055701 (2013). 
[40] J. Bialké, H. Löwen, and T. Speck, Europhys. Lett. 103, 30008 (2013).

[41] T. F. F. Farage, P. Krinninger, and J. M. Brader, Phys. Rev. E 91, 042310 (2015).

[42] J. T. Siebert, J. Letz, T. Speck, and P. Virnau, Soft Matter 13, 1020 (2017).

[43] P. Digregorio, D. Levis, A. Suma, L. F. Cugliandolo, G. Gonnella, and I. Pagonabarraga, Phys. Rev. Lett. 121, 098003 (2018).

[44] D. Loi, S. Mossa, and L. F. Cugliandolo, Phys. Rev. E 77, 051111 (2008).

[45] D. Levis and L. Berthier, Europhys. Lett. 111, 60006 (2015).

[46] G. Szamel, Phys. Rev. E 90, 012111 (2014).

[47] L. F. Cugliandolo, G. Gonnella, and I. Petrelli, Fluctuation Noise Lett. 18, 1940008 (2019).

[48] É. Fodor, C. Nardini, M. E. Cates, J. Tailleur, P. Visco, and F. van Wijland, Phys. Rev. Lett. 117, 038103 (2016).

[49] G. Szamel, Europhys. Lett. 117, 50010 (2017).

[50] K. Asheichyk, A. P. Solon, C. M. Rohwer, and M. Krüger, J. Chem. Phys. 150, 144111 (2019).

[51] P. B. Warren and R. J. Allen, Phys. Rev. Lett. 109, 250601 (2012).

[52] C. Gardiner, Stochastic Methods (Springer, Berlin, 2009).

[53] J. Tailleur and M. E. Cates, Phys. Rev. Lett. 100, 218103 (2008).

[54] M. E. Cates and J. Tailleur, Europhys. Lett. 101, 20010 (2013).

[55] A. P. Solon, M. Cates, and J. Tailleur, Eur. Phys. J. Spec. Top. 224, 1231 (2015).

[56] L. L. Bonilla, Phys. Rev. E 100, 022601 (2019).

[57] The generalized potential is related to the information potential in stochastic thermodynamics (see Ref. [8]).

[58] L. Dabelow, S. Bo, and R. Eichhorn, Phys. Rev. X 9, 021009 (2019).
[59] T. Hatano and S.-i. Sasa, Phys. Rev. Lett. 86, 3463 (2001).

[60] A. Ghosh, J. Samuel, and S. Sinha, Europhys. Lett. 98, 30003 (2012).

[61] The reduced ABP model can be mapped into AOUP by identifying $v_{0}^{2} / 2$ in ABP with $D / \tau$ in AOUP. However, in order to recover the passive limit, translational thermal noise has to be included in ABP while, for AOUP, Gaussian white noise is recovered in the $\tau \rightarrow 0$ limit in the absence of translational noise because of the $1 / \tau$ factor in the noise variance.

[62] P. Hänggi and P. Jung, Adv. Chem. Phys. 89, 239 (1994).

[63] S. Adelman, J. Chem. Phys. 64, 124 (1976).

[64] M. San Miguel and J. Sancho, J. Stat. Phys. 22, 605 (1980).

[65] R. F. Fox, Phys. Rev. A 34, 4525 (1986).

[66] R. F. Fox, Phys. Rev. A 33, 467 (1986).

[67] R. Wittmann, C. Maggi, A. Sharma, A. Scacchi, J. M. Brader, and U. M. B. Marconi, J. Stat. Mech. (2017) 113207.

[68] U. M. B. Marconi and C. Maggi, Soft Matter 11, 8768 (2015).

[69] L. Caprini, U. M. B. Marconi, and A. Vulpiani, J. Stat. Mech. (2018) 033203.

[70] F. J. Sevilla, A. V. Arzola, and E. P. Cital, Phys. Rev. E 99, 012145 (2019).

[71] N. G. van Kampen, J. Stat. Phys. 54, 1289 (1989).

[72] S. Hanna, W. Hess, and R. Klein, J. Phys. A 14, L493 (1981).

[73] J. Palacci, C. Cottin-Bizonne, C. Ybert, and L. Bocquet, Phys. Rev. Lett. 105, 088304 (2010).

[74] F. Ginot, I. Theurkauff, D. Levis, C. Ybert, L. Bocquet, L. Berthier, and C. Cottin-Bizonne, Phys. Rev. X 5, 011004 (2015).

[75] C. Chatelain, J. Phys. A 36, 10739 (2003).

[76] P. Warren and R. Allen, Entropy 16, 221 (2014).

[77] A. Nikoubashman and T. Ihle, Phys. Rev. E 100, 042603 (2019). 